\title{
Identidad digital y conectividad: conocimiento y actitudes en estudiantes universitarios chilenos
}

\author{
Marisol P. Hernández-Orellana1, Adolfina Pérez-Garcias ${ }^{2}$, y Ángel G. Roco-Videla ${ }^{3,4^{*}}$ \\ (1) Unidad de Desarrollo Corporativo, Informática educativa, Universidad Autónoma de Chile, Galvarino Gallardo 1983. \\ Santiago-Chile (correo-e: marisol.hernandez@uautonoma.cl). \\ (2) Departamento de Pedagogía Aplicada y Psicología de Educación, Universidad de las Islas Baleares, Carretera de \\ Valldemossa, km 7.5, 07122 Palma, Illes Balears-España (correo-e: fina.perez@uib.es). \\ (3) Facultad de Salud, programa Magíster en Ciencias Químico-Biológicas, Universidad Bernardo O’Higgins, General \\ Gana 1702, Santiago-Chile (correo-e: angel.roco.videla@gmail.com). \\ (4) Facultad de ingeniería, Departamento de Ingeniería Civil, Universidad Católica de la Santísima Concepción, Alonso de \\ Rivera 2850, Concepción-Chile (correo-e: aroco@ucsc.cl).
}

* Autor a quien debe ser dirigida la correspondencia

Recibido Jun. 9, 2020; Aceptado Ago. 7, 2020; Versión final Sep. 9, 2020, Publicado Feb. 2021

\begin{abstract}
Resumen
El objetivo de esta investigación fue determinar el nivel de conocimiento y las actitudes de estudiantes universitarios chilenos frente a los conceptos de identidad y reputación digital y sus hábitos de conectividad a medios digitales. Se utilizó el área de formación (ciencias de la ingeniería, ciencias de la salud y humanidades) como eje de análisis. La investigación fue descriptiva-cuantitativa y de corte transversal. Se aplicó un instrumento previamente validado a una muestra de 509 voluntarios agrupados en tres áreas: ciencias de la ingeniería, ciencias de la salud y humanidades. Se utilizó la prueba Kruskal Wallis para el análisis estadístico. Los resultados muestran que el celular fue el dispositivo preferente de acceso a Internet. Los estudiantes no hacen diferencias entre su yo presencial y su yo digital. Se concluye que el área de formación no influye en la elección del tipo, ni tiempo de conexión, ni dispositivo preferente, pero sí en su concepto de reputación digital y su gestión.
\end{abstract}

Palabras clave: identidad digital; conectividad; reputación; interacción interpersonal; ningufoneo

\section{Digital identity and connectivity: knowledge and attitudes of Chilean university students}

\begin{abstract}
The objective of this research study was to determine the knowledge level and the attitudes of Chilean university students towards the concepts of identity and digital reputation and their connectivity habits to digital media. The subject area (engineering, health sciences, and humanities) of study was used as the analysis axis. The research was descriptive-quantitative and cross-sectional. A previously validated instrument was applied to a sample of 509 volunteers grouped in three areas: engineering sciences, health sciences, and humanities. The Kruskal Wallis test was used for statistical analysis. The results showed that cellphones were the preferred device to access the Internet. Students did not differentiate between their face self and their digital self. It is concluded that the subject area of study did not influence choice type, connection time, or preferred device, but it did influence student's digital reputation concept and management.
\end{abstract}




\section{INTRODUCCIÓN}

La sociedad de la información en la que vivimos se ha caracterizado por una rápida obsolescencia del conocimiento debido al aumento en el uso sistemático de las tecnologías de la información y comunicación (TIC); transformándola en un escenario dinámico, variable y altamente competitivo en el que las esferas de acción e interacción han mutado y, por consiguiente, la construcción de la identidad también se ha visto afectada (Castro y González, 2016; Basantes et al, 2017). La identidad personal se construye no solo en la medida en que las personas (usuarios en la red) se revelan, sino también en función del grado de disponibilidad con y entre sus contactos (presencia), por el nivel de interacción e intercambio de contenido con otros (compartir), por la riqueza e interactividad con que se relaciona con otros (relación), por la intensidad y complejidad de la red de usuarios comunicados entre sí (conversar), por el tipo de opiniones sobre la situación social y de los contenidos que se publican (reputación) y, finalmente, por el grado o medida en que los usuarios son etiquetados o forman parte de comunidades (grupos) (Kietzmann et al, 2011).

En Chile el acceso a internet, es decir, la posibilidad de conexión a una red digital ya sea a través de una dispositivo móvil o fijo ha sido creciendo rápidamente, ya en el año 2017 85.7\% de las personas tienen acceso a internet. El uso de internet, es decir, la actividad que se lleva a cabo cuando se acceder a la red, ha ido también presentando cambios con un aumento en cuanto a la comunicación con otros a través de medios digitales, pasando de un 68,9\% en el 2016 a un 78,1\% en el 2017 y la búsqueda de información que paso de un $66,2 \%$ en el 2016 a un 73,7\% en el 2017 (Subsecretaria de Telecomunicaciones, 2018). Cuando hacemos uso de Internet vamos dejando huellas digitales que van definiendo y mostrando nuestra identidad digital, proceso que para las nuevas generaciones deviene desde la infancia. Lo sempiterno de dichas huellas las tornan casi imposibles de borrar y difícilmente se pueden ocultar (Feher, 2019; Brandtzaeg, 2020). Con el paso del tiempo estas se vuelven más profusas e intensas gracias al constante acceso a Internet por medio de diferentes dispositivos con los que interactuamos a diario. Los teléfonos inteligentes, notebook, consolas de videojuegos, entre otros, se han transformado en parte esencial de la vida cotidiana, en especial, para los más jóvenes (Hilt et al, 2019; Barrios et al, 2018).

En los medios digitales los usuarios tienen control de aproximadamente del $70 \%$ de su huella digital, lo que implica que el $30 \%$ de las actividades en línea están inconscientemente flotando con dinámicas digitales. Esto da como resultado una amplia gama de consecuencias no esperadas como robos de identidad, entre muchas otras (Feher, 2019). Comprender el impacto a largo plazo de acciones tan simples como compartir una fotografía, para la propia reputación, es difícil de asimilar para las nuevas generaciones. Ello, puesto que estas experiencias no han sido vividas por sus padres o generaciones anteriores, que puedan guiarlos con el ejemplo (Williams at al; 2013).

Por tanto, quienes han crecido con internet son más propensos a compartir información personal y confidencial en línea, lo cual no quiere decir que no les preocupe su privacidad; de hecho, son conscientes de los riesgos que implica el mal uso de su información personal, en relación con su propia reputación y su seguridad, pero eso no impide que la compartan (Holicza et al., 2018). Más aun considerando que los teléfonos inteligentes, como herramientas indispensables para cubrir necesidades profesionales y sociales, desde un solo dispositivo, permiten una presencia virtual omnipresente (Soomro et al., 2019). Esto ha generado una creciente necesidad por estar conectados cada vez más tiempo, desembocando en dependencia y/o adicción (Kundapur et al., 2020); siendo la manifestación más frecuente entre adolescentes y adultos jóvenes de esta adición el ningufoneo o phubbing (en inglés). Este nuevo concepto apunta al fenómeno social de mirar constantemente un dispositivo móvil mientras se interactúa con otros de manera presencial con lo que se perturba, finalmente, la conversación y comunicación (Liu et al., 2019; Vanden et al, 2019).

Esto marca una diferencia significativa en la forma en que este grupo etario ha construido su identidad digital en comparación con quienes no han crecido en un ambiente altamente digitalizado (Yu, 2020; Kurek, 2017). La reputación digital, al igual que la identidad digital, se construye a través de un proceso narrativo que conlleva una serie de acciones en el tiempo y que es el resultado de la combinación del mundo real (que preferimos llamar presencial, porque todos son reales) y digital (Cöteli et al, 2019; Stuart et al, 2019; Addallah, 2018). Para la Dra. Fanny Georges (2012), la construcción de la identidad digital implica no solo los imperativos que puede o no declarar el individuo en la red, sino que también, en la Web 2.0 el usuario que desea existir debe cumplir con la exigencia de producir actividades continuamente las cuales el sistema, por sí mismo, evalúa afectando su actividad, su reputación e identidad digital (Castro et al, 2016).

Todo lo anterior ha implicado una transformación en los procesos de enseñanza y aprendizaje (López et al, 2016) y un desafío importante para las Instituciones de educación superior, las que deben dar respuesta a nuevos requerimientos, instándolas a la formación de profesionales digitalmente autónomos que actualicen permanentemente sus conocimientos y competencias para hacer frente a las necesidades actuales y futuras 
(Basantes et al, 2017). Para lograr esta transformación en el modelo educativo actual es, imprescindible, conocer cómo los estudiantes perciben este impacto tecnológico en su propia construcción identitaria en relación con la información, cómo acceden a ella y los factores que pueden influir en estos aspectos.

El objetivo de esta investigación fue determinar el nivel de conocimiento y actitudes frente a los conceptos de identidad y reputación digital, así como sus hábitos de conectividad a medios digitales, utilizando el área de formación como eje de análisis y como parte de los componentes fundamentales que permiten la construcción de la identidad digital en una muestra de estudiantes pertenecientes a 21 universidades chilenas participantes, que se encuentran tanto en la Región Metropolitana, como centro y sur del país.

\section{METODOLOGÍA}

La investigación realizada fue de tipo descriptiva de corte transversal bajo el paradigma cuantitativo. Los resultados presentados en este articulo fueron parte de la información obtenida en la realización de la tesis para obtención del grado de Doctor en Tecnologías Educativas.

Para determinar el tamaño de la muestra a estudiar se consideró la población como de tamaño infinito o desconocido y se utilizó la fórmula para una proporción (Garcia-Garcia et al; 2013), obteniendo un número de 384 sujetos, siendo este el número mínimo que permitiría tener una muestra representativa de la población. Se evaluaron finalmente un total de 509 sujetos voluntarios pertenecientes a 21 universidades de un total 55 universidades en funcionamiento sin proceso de cierre vigente (Ministerio de Educación, 2020). Esta muestra se dividió en 132 estudiantes universitarios de carreras asociadas al área de Ciencias de la Ingeniería, 224 al área de Ciencias de la Salud y 153 al área de Humanidades. El proceso de recolección de información duró tres meses y todos los sujetos participaron de manera voluntaria acorde al consentimiento informado. Como criterios de inclusión se consideraron solo individuos mayores de 18 años (mayoría de edad legal en Chile) y que cursarán una carrera en modalidad presencial diurna.

El instrumento utilizado constó de 2 preguntas de selección múltiple y 10 preguntas en escala tipo Likert subdivido en diferentes afirmaciones asociadas a cada pregunta. La validación de este cuestionario fue a partir del juicio de expertos utilizando la técnica de grupo nominal (Cabero y Llorente, 2013), dicho grupo estuvo conformado por 12 doctores pertenecientes a universidades europeas y latinoamericanas, divididos en dos grupos: a) con publicaciones en identidad digital y b) con publicaciones en tecnología educativa.

El análisis de los resultados obtenidos fue de tipo descriptivo, utilizando la distribución porcentual de las respuestas. Para establecer si existía diferencia significativa entre las respuestas de cada grupo, se utilizó la prueba Kruskal Wallis, correspondiente para un método no paramétrico, donde $\mathrm{H}_{0}$ nos señala que no hay diferencias entre los grupos y $\mathrm{H}_{\mathrm{A}}$ nos indica que al menos uno de los grupos es diferente. Se rechazará $\mathrm{H}_{0}$ cuando el valor de $p$ obtenido sea menor o igual a 0.05 . Esta prueba se trabajó con un $95 \%$ de confianza, utilizando el programa estadístico Stata en su versión 14, en español.

\section{RESULTADOS Y DISCUSIÓN}

En las siguientes tablas se resumen los resultados obtenidos expresados como porcentajes que ilustran la distribución de las respuestas obtenidas por cada grupo de estudiante según el área de formación. En cuanto a las diferencias por grupo, en todos los casos cuando el valor $p$ obtenido es menor o igual a 0.05 se considerará que existe una diferencia significativa en, al menos uno, de los grupos respecto al resto.

En la tabla 1 se presentan las respuestas en relación con el conocimiento de los estudiantes según área de formación respecto a los conceptos de identidad y reputación digitales. Cerca del $40 \%$ de los estudiantes en promedio comprenden lo que implica el concepto de identidad digital, no existiendo diferencias por área de formación (valor- $p=0.706$ ). El concepto de reputación digital es mucho más conocido por los estudiantes y en promedio un $67.19 \%$ es capaz de identificar correctamente su definición. No obstante, un $31.06 \%$ de los estudiantes del área de Ciencias de la Ingeniería asocia este concepto a la manera particular en la que cada persona se autogestiona en la Web, el conocimiento que ha adquirido sobre las TIC, es decir, ven la reputación digital como la forma en que las personas gestionan sus conocimientos. En este caso si se encontraron diferencias significativas por área siendo el área, siendo el área Ciencias de la Ingeniería la que presenta el menor porcentaje de respuestas correctas en cuanto a la definición de lo que es e implica la reputación digital (valor- $p=0.002$ ).

En cuanto a la preocupación en relación con su identidad digital, en la tabla 2 se puede observar que no es un aspecto que represente una gran preocupación. En las tres áreas los niveles Poco y Algo acumulan más del $55 \%$ de las respuestas. Lo mismo ocurre respecto a la pregunta de que si se han buscado en Internet, donde las opciones Pocas y Algunas veces acumulan en todos los casos más de $55 \%$ del total de respuestas. 
Tabla 1: Conocimiento respecto a los conceptos de reputación e identidad digital.

\begin{tabular}{|c|c|c|c|}
\hline \multicolumn{4}{|c|}{ ¿Cuál de las siguientes afirmaciones crees que representa mejor el concepto de identidad digital? } \\
\hline & Cs. Ing. & Cs. Salud & Humanidades \\
\hline $\begin{array}{l}\text { A.- Nombre virtual con que se conoce a una persona en Internet y que } \\
\text { es distinto al que usa en la presencialidad. }\end{array}$ & $25.76 \%$ & $28.57 \%$ & $21.57 \%$ \\
\hline $\begin{array}{l}\text { B.- El rastro que cada usuario de Internet deja en la red como } \\
\text { resultado de su interacción con otros usuarios. }\end{array}$ & $21.97 \%$ & $18.75 \%$ & $20.92 \%$ \\
\hline $\begin{array}{l}\text { C.- Complejo conjunto de datos e información formado por todo lo que } \\
\text { decimos y hacemos en Internet y, también, por lo que los demás dicen } \\
\text { que hacemos o decimos }\left(^{* *}\right) \text {. }\end{array}$ & $38.64 \%$ & $37.05 \%$ & $45.10 \%$ \\
\hline $\begin{array}{l}\text { D.- Competencia tecnológica que demuestra la manera particular en la } \\
\text { que cada persona autogestiona, en la web, el conocimiento que ha } \\
\text { adquirido sobre las TIC. }\end{array}$ & $11.36 \%$ & $3.13 \%$ & $5.88 \%$ \\
\hline E.- No conozco el concepto. & $2.27 \%$ & $12.50 \%$ & $6.54 \%$ \\
\hline \multicolumn{4}{|l|}{ Valor $-p=0.706$} \\
\hline \multicolumn{4}{|c|}{ ¿Cuál de las siguientes afirmaciones representa mejor el concepto de reputación digital? } \\
\hline $\begin{array}{l}\text { A.- Opinión crítica que se tiene sobre una persona respecto de su } \\
\text { manejo tecnológico. }\end{array}$ & $8.33 \%$ & $8.48 \%$ & $8.50 \%$ \\
\hline $\begin{array}{l}\text { B.- Interpretación que otros se hacen del individuo a partir de la } \\
\text { información que éste deja en la red o de la que otros publican sobre } \\
\text { él. }\left(^{* *}\right)\end{array}$ & $53.79 \%$ & $75.89 \%$ & $71.90 \%$ \\
\hline $\begin{array}{l}\text { C.- La manera particular en la que cada persona autogestiona, en la } \\
\text { web, el conocimiento que ha adquirido sobre las TIC. }\end{array}$ & $31.06 \%$ & $6.70 \%$ & $11.76 \%$ \\
\hline D.- No conozco el concepto & $6.82 \%$ & $8.93 \%$ & $7.84 \%$ \\
\hline \multicolumn{4}{|c|}{ Valor- $p=0.002\left(^{*}\right) \quad\left(^{*}\right)$ Significancia $p<0.05 ;\left(^{* *}\right)$ Respuesta correcta (Magro, 2016) } \\
\hline
\end{tabular}

En cuanto el hecho de ocuparse de la gestión de su reputación digital, los estudiantes de Ciencias de la Ingeniería mostraron un mayor interés, pero esto se puede deber también a que un alto porcentaje de ellos entiende la reputación digital como autogestión del conocimiento en la Web, lo cual puede ser la causa de la diferencia observada.

Tabla 2: Actitudes frente a la identidad digital: Preocupación, auto búsqueda y gestión de reputación

\begin{tabular}{|c|c|c|c|c|c|c|}
\hline \multicolumn{7}{|c|}{ ¿Te preocupa tu identidad digital? } \\
\hline & Nada & Poco & Algo & Bastante & Mucho & Valor- $p$ \\
\hline Cs. Ing. & $8.33 \%$ & $24.24 \%$ & $31.82 \%$ & $27.27 \%$ & $8.33 \%$ & \multirow{3}{*}{0.108} \\
\hline Cs. Salud & $12.95 \%$ & $26.34 \%$ & $35.71 \%$ & $19.64 \%$ & $5.36 \%$ & \\
\hline Humanidades & $14.38 \%$ & $26.14 \%$ & $33.33 \%$ & $14.38 \%$ & $11.76 \%$ & \\
\hline \multicolumn{7}{|c|}{ ¿Te has buscado a ti mismo alguna vez en internet? } \\
\hline & Nunca & Pocas veces & Algunas veces & Varias veces & Muchas veces & Valor- $p$ \\
\hline Cs. Ing. & $15.15 \%$ & $36.36 \%$ & $25.76 \%$ & $13.64 \%$ & $9.09 \%$ & \multirow{3}{*}{0.197} \\
\hline Cs. Salud & $18.30 \%$ & $41.07 \%$ & $25.89 \%$ & $10.71 \%$ & $4.02 \%$ & \\
\hline Humanidades & $21.57 \%$ & $35.29 \%$ & $25.49 \%$ & $9.15 \%$ & $8.50 \%$ & \\
\hline \multicolumn{7}{|c|}{ ¿Te ocupas de gestionar tu reputación digital? } \\
\hline & Nada & Poco & Algo & Bastante & Mucho & Valor- $p$ \\
\hline Cs. Ing. & $20.45 \%$ & $23.48 \%$ & $28.79 \%$ & $18.18 \%$ & $9.09 \%$ & \multirow{3}{*}{$0.004\left(^{*}\right)$} \\
\hline Cs. Salud & $32.14 \%$ & $28.13 \%$ & $21.88 \%$ & $16.96 \%$ & $0.89 \%$ & \\
\hline Humanidades & $26.80 \%$ & $30.72 \%$ & $23.53 \%$ & $14.38 \%$ & $4.58 \%$ & \\
\hline$\left(^{*}\right)$ Significanc & 0.05 & & & & & \\
\hline
\end{tabular}


En la tabla 3 se analiza la actitud frente a la identidad digital en relación con la similitud que los estudiantes perciben entre su yo presencial y digital, es decir, su yo dentro y fuera de las redes; aquí se puede observar que no se encontraron diferencias significativas entre las tres áreas de formación (valor- $p=0.297$ ). Si se observa la distribución porcentual, los valores 8, 9 y 10 acumulan en todos los casos se aproxima a un $70 \%$ de las preferencias expresadas por los estudiantes. En general, la distribución de las respuestas apunta al hecho de que los estudiantes de esta muestra no hacen diferencias entre su yo presencial y digital. También es posible hace una relación entre la nota promedio por área con el porcentaje de similitud entre su yo presencial y digital, dado que la escala es de 1 a 10 esta es equiparable a la escala de 1 a 100 de los porcentajes. Así, podemos decir que en el caso de los estudiantes de Ingeniería la similitud llegaría a un $81.8 \%$, en el caso de los estudiantes de Ciencias de la Salud esta llegaría a un $79.7 \%$ y para los estudiantes del área de Humanidades la similitud sería de un $78.2 \%$.

Tabla 3: Actitudes frente a la identidad digital: Similitud entre el yo presencial y el digital

\begin{tabular}{|c|c|c|c|c|c|c|c|c|c|c|}
\hline \multicolumn{11}{|c|}{$\begin{array}{l}\text { Cuánto se parece el "yo" que expresas en las redes sociales a tu "yo" fuera de ellas. Valora de } 1 \text { a } 10 \text {, siendo } 1 \text { la } \\
\text { menor coincidencia y } 10 \text { la mayor coincidencia. }\end{array}$} \\
\hline & 1 & 2 & 3 & 4 & 5 & 6 & 7 & 8 & 9 & 10 \\
\hline Cs. Ing. & $0.76 \%$ & $0.76 \%$ & $2.27 \%$ & $2.27 \%$ & $5.30 \%$ & $4.55 \%$ & $11.36 \%$ & $24.24 \%$ & $12.12 \%$ & $36.36 \%$ \\
\hline Cs. Salud & $1.79 \%$ & $0.45 \%$ & $2.23 \%$ & $1.79 \%$ & $6.70 \%$ & $7.14 \%$ & $9.38 \%$ & $24.55 \%$ & $17.41 \%$ & $28.57 \%$ \\
\hline Humanidades & $2.61 \%$ & $0.65 \%$ & $2.61 \%$ & $1.31 \%$ & $7.84 \%$ & $5.23 \%$ & $10.46 \%$ & $26.80 \%$ & $17.65 \%$ & $24.84 \%$ \\
\hline \multicolumn{2}{|c|}{ Promedio Cs. Ing. } & \multicolumn{2}{|c|}{8.18} & \multicolumn{2}{|c|}{ Promedio Cs. Salud } & \multicolumn{2}{|c|}{7.97} & \multicolumn{2}{|c|}{ Promedio Humanidades } & 7.82 \\
\hline \multicolumn{2}{|c|}{ Valor- $p$} & \multicolumn{9}{|c|}{0.2397} \\
\hline \multicolumn{11}{|c|}{ Significancia: $p<0.05$} \\
\hline
\end{tabular}

En la tabla 4 se analizan las actitudes frente a la identidad digital, donde se puede observar que los estudiantes presentan una mayor preferencia a la conversación en forma presencial cuando consideran que el tema a tratar es de importancia. En segunda instancia, se presenta el correo electrónico y dejando como opción final la llamada telefónica. Es interesante observar que al ser la conversación presencial la primera preferencia, el uso de la video conferencia no fuera la segunda, sino la tercera opción, a pesar de poder considerarse una alternativa mucho más cercana a la presencialidad, permitiendo no solo compartir el lenguaje verbal, sino que también el no verbal. Pese a ello, esta alternativa solo llegó a un $21.86 \%$ promedio de primera preferencia, contra un $25.72 \%$ del uso del correo electrónico.

En cuanto a la interacción en Internet se puede observar una clara relación entre el grado de importancia que le dan al otro u otros con los cuales están interactuando y el hecho de ingresar o no a Internet en su presencia. Es así que, cuando están solos ven validada la opción de ingresar a la red, no hay personas que objeten dicha conducta y se convierte en la primera preferencia, con un promedio de $87.52 \%$ entre las tres áreas. Este porcentaje comienza a bajar cuando se está con amigos, llegando a un $49.66 \%$ promedio versus cuando se está en clases, que el promedio llega a un $39.46 \%$. Cuando los estudiantes se encuentran a un espacio de mayor cercanía e intimidad, como lo es compartiendo con la familia o la pareja, se produce una baja considerable en los porcentajes promedios de interacción en Internet, siendo de un $23.72 \%$ al estar con la familia y un $20.62 \%$ cuando se interactúa con la pareja.

Si bien el estar solo es la instancia donde se suele aumentar la interacción mucho más en Internet, se encontraron diferencias significativas por área de formación (valor- $p=0.001$ ), siendo los estudiantes de Ciencias de la Ingeniería quienes presentaron el menor porcentaje en esta afirmación con un $81.06 \%$. Al mismo tiempo este grupo presentó el menor uso de Internet estando en clases y el mayor porcentaje cuando se estaba con la pareja o con sus familias en relación con las otras dos áreas de formación; aunque estas diferencias no llegaron a ser estadísticamente significativas. En la última sección de la tabla 4 se consulta respecto al plano o ambiente donde los estudiantes se sienten con mayor libertad para expresar su identidad, en este caso no se encontraron tampoco diferencias según área de formación. El plano presencial lidera las preferencias con un $52.37 \%$ promedio entre las tres áreas, seguido por la opción ambas (virtual-presencial) con un $37.28 \%$ y el ambiente virtual solo alcanzó un $10.37 \%$ promedio.

En la tabla 5 se realiza un análisis de la conectividad donde se evidencia que para acceder a Internet los estudiantes encuestados prefieren hacerlo por medio del plan de datos del celular con un $78.03 \%$ promedio de preferencia entre las tres áreas. El teléfono móvil es el equipo preferente de uso con un $89.70 \%$ promedio. Con respecto al uso de PC de escritorio, se encontraron diferencias significativas entre los grupos (valor$p=0.001$ ) donde quienes hacen un mayor uso son los estudiantes del área de Humanidades. En promedio, el $51.28 \%$ de los estudiantes de las tres áreas está más de 4 horas activo en la red al día, siendo los estudiantes de Ciencias de la Salud los que presentan el mayor porcentaje con un $55.36 \%$. 
Tabla 4: Actitudes frente a la identidad digital: Interacciones

\begin{tabular}{|c|c|c|c|c|c|c|c|c|}
\hline \multicolumn{4}{|c|}{ ¿Si debes conversar algo importante prefieres hacerlo } & \multicolumn{5}{|c|}{1 corresponde al que más usarías y 5 al que menos. } \\
\hline & & & 1 & 2 & 3 & 4 & 5 & Valor-p \\
\hline \multirow[t]{3}{*}{ Presencialmente } & \multicolumn{2}{|c|}{ Cs. Ing. } & $46.21 \%$ & $9.85 \%$ & $3.03 \%$ & $3.79 \%$ & $37.12 \%$ & \multirow{3}{*}{0.109} \\
\hline & \multicolumn{2}{|c|}{ Cs. Salud } & $40.18 \%$ & $0.45 \%$ & $3.57 \%$ & $4.46 \%$ & $51.34 \%$ & \\
\hline & \multicolumn{2}{|c|}{ Humanidades } & $43.79 \%$ & $1.96 \%$ & $1.96 \%$ & $5.23 \%$ & $47.06 \%$ & \\
\hline \multirow[t]{3}{*}{ Por mensaje de texto } & \multicolumn{2}{|c|}{ Cs. Ing. } & $14.39 \%$ & $21.21 \%$ & $27.27 \%$ & $20.45 \%$ & $16.67 \%$ & \multirow{3}{*}{0.154} \\
\hline & \multicolumn{2}{|c|}{ Cs. Salud } & $4.91 \%$ & $21.43 \%$ & $31.70 \%$ & $30.36 \%$ & $11.61 \%$ & \\
\hline & \multicolumn{2}{|c|}{ Humanidades } & $13.07 \%$ & $19.61 \%$ & $33.33 \%$ & $22.88 \%$ & $11.11 \%$ & \\
\hline \multirow[t]{3}{*}{ Telefónicamente } & \multicolumn{2}{|c|}{ Cs. Ing. } & $5.30 \%$ & $25.00 \%$ & $40.91 \%$ & $17.42 \%$ & $11.36 \%$ & \multirow{3}{*}{0.849} \\
\hline & \multicolumn{2}{|c|}{ Cs. Salud } & $4.02 \%$ & $32.25 \%$ & $34.82 \%$ & $23.21 \%$ & $6.70 \%$ & \\
\hline & \multicolumn{2}{|c|}{ Humanidades } & $9.15 \%$ & $30.07 \%$ & $22.88 \%$ & $28.10 \%$ & $9.80 \%$ & \\
\hline \multirow[t]{3}{*}{ Por videoconferencia } & \multicolumn{2}{|c|}{ Cs. Ing. } & $9.85 \%$ & $31.82 \%$ & $20.45 \%$ & $21.97 \%$ & $15.91 \%$ & \multirow{3}{*}{0.179} \\
\hline & \multicolumn{2}{|c|}{ Cs. Salud } & $25.00 \%$ & $22.32 \%$ & $15.18 \%$ & $25.45 \%$ & $12.05 \%$ & \\
\hline & \multicolumn{2}{|c|}{ Humanidades } & $30.72 \%$ & $11.76 \%$ & $13.07 \%$ & $16.99 \%$ & $27.45 \%$ & \\
\hline \multirow[t]{3}{*}{ Por correo electrónico } & \multicolumn{2}{|c|}{ Cs. Ing. } & $21.21 \%$ & $12.88 \%$ & $10.61 \%$ & $18.94 \%$ & $36.36 \%$ & \multirow{3}{*}{0.069} \\
\hline & \multicolumn{2}{|c|}{ Cs. Salud } & $30.36 \%$ & $16.96 \%$ & $10.71 \%$ & $12.05 \%$ & $29.91 \%$ & \\
\hline & \multicolumn{2}{|c|}{ Humanidades } & $25.58 \%$ & $33.73 \%$ & $36.67 \%$ & $36.59 \%$ & $25.81 \%$ & \\
\hline La mayoría c & las & es en qu & stás inter & uando en & ernet lo $\mathrm{h}$ & es cuand & e encuent & \\
\hline & & & Cs. Ing. & $\mathrm{Cs}$ & alud & Hur & nidades & Valor-p \\
\hline Conversando con amig & & No & $50.00 \%$ & & $4 \%$ & & $67 \%$ & 0.943 \\
\hline & & $\mathrm{Si}$ & $50.00 \%$ & & $6 \%$ & & $33 \%$ & \\
\hline Compartiendo con la pa & & No & $75.00 \%$ & & $0 \%$ & & $35 \%$ & 0.268 \\
\hline & & $\mathrm{Si}$ & $25.00 \%$ & & $0 \%$ & & $65 \%$ & \\
\hline Compartiendo con la fa & & No & $75.00 \%$ & & $1 \%$ & & $74 \%$ & 0.431 \\
\hline & & $\mathrm{Si}$ & $25.00 \%$ & & $9 \%$ & & $26 \%$ & \\
\hline Estando en clases & & No & $62.12 \%$ & & $8 \%$ & & $13 \%$ & 0.876 \\
\hline (escuchando/participan & & $\mathrm{Si}$ & $37.88 \%$ & & $3 \%$ & & $87 \%$ & \\
\hline Estando solo & & No & $18.94 \%$ & & $4 \%$ & & $46 \%$ & $0.001\left(^{*}\right)$ \\
\hline & & $\mathrm{Si}$ & $81.06 \%$ & & $6 \%$ & & $54 \%$ & \\
\hline ¿En cuál de los sigu & & nos o an & entes sien & que tiene & nayor lib & d para ex & esar de tı & ntidad? \\
\hline & & & Ing. & & Salud & $\mathrm{Hur}$ & nidades & Valor- $p$ \\
\hline Presencial & & & $73 \%$ & & $13 \%$ & & $21 \%$ & \\
\hline Virtual (no presencial) & & & $51 \%$ & & $38 \%$ & & $11 \%$ & 0.296 \\
\hline En ambas por igual & & & $37 \%$ & & $50 \%$ & & $68 \%$ & \\
\hline (*) Significancia: $p<0$. & & & & & & & & \\
\hline
\end{tabular}

Tabla 5: Conectividad según área de formación

\begin{tabular}{|c|c|c|c|c|c|c|c|c|c|}
\hline \multicolumn{10}{|c|}{ Desde qué tipo de conexión accedes a internet. La que más usas (2-3) hasta la que menos usas o no usas (0-1) } \\
\hline & \multicolumn{2}{|c|}{ Wifi gratuito } & \multicolumn{2}{|c|}{ Wifi de tu casa } & \multicolumn{3}{|c|}{ Internet del celular } & \multicolumn{2}{|c|}{ Banda ancha móvil } \\
\hline & $0-1$ & $2-3$ & $0-1$ & $2-3$ & \multicolumn{2}{|c|}{$0-1$} & $2-3$ & $0-1$ & $2-3$ \\
\hline Cs. Ing. & $68.18 \%$ & $31.82 \%$ & $21.21 \%$ & $78.79 \%$ & \multicolumn{2}{|c|}{$21.97 \%$} & $78.03 \%$ & $84.09 \%$ & $15.91 \%$ \\
\hline Cs. Salud & $70.09 \%$ & $29.91 \%$ & $26.79 \%$ & $79.92 \%$ & \multicolumn{2}{|c|}{$23.66 \%$} & $76.34 \%$ & $87.50 \%$ & $12.50 \%$ \\
\hline Humanidades & $66.01 \%$ & $33.99 \%$ & \multirow{2}{*}{\multicolumn{2}{|c|}{\begin{tabular}{c|c}
$28.76 \%$ & $71.25 \%$ \\
0.193
\end{tabular}}} & 20.26 & $6 \%$ & $79.74 \%$ & $85.62 \%$ & $14.38 \%$ \\
\hline Valor- $p$ & \multicolumn{2}{|c|}{0.711} & & & \multicolumn{3}{|c|}{0.928} & \multicolumn{2}{|c|}{0.502} \\
\hline \multirow{2}{*}{\multicolumn{8}{|c|}{\begin{tabular}{|c|c}
\multicolumn{2}{|c|}{ Dispositivos del cual interactúas en in } \\
\end{tabular}}} & \multicolumn{2}{|c|}{ usas. (0-1) } \\
\hline & & Notebook & \multicolumn{2}{|c|}{ teléfono móvil } & \multicolumn{3}{|r|}{ Tablet } & \multicolumn{2}{|c|}{ PC escritorio } \\
\hline & $0-1$ & $2-3$ & $0-1$ & $2-3$ & \multicolumn{2}{|r|}{$0-1$} & $2-3$ & $0-1$ & $2-3$ \\
\hline Cs. Ing. & $25,00 \%$ & $75.00 \%$ & $15.15 \%$ & $84.88^{\circ}$ & & $81.06 \%$ & 18.94 & $75.00 \%$ & $25.00 \%$ \\
\hline Cs. Salud & $20.54 \%$ & $79.46 \%$ & $12.06 \%$ & $97.94^{\circ}$ & & $82.59 \%$ & 17.41 & $79.92 \%$ & $20.08 \%$ \\
\hline Humanidades & $30.06 \%$ & $69.94 \%$ & $13.73 \%$ & $86.27^{\circ}$ & & $86.28 \%$ & 13.73 & $67.97 \%$ & $32.03 \%$ \\
\hline Valor-p & & 77 & & 0.316 & & & 0.253 & & $1\left(^{*}\right)$ \\
\hline & & & Cuántas r & oras estas a & ctivo er & n la red & & & \\
\hline & $\begin{array}{r}\text { Menos } \\
\text { hora a }\end{array}$ & & $\begin{array}{l}\text { Más } 1 \text { hasta } \\
\text { horas al día }\end{array}$ & $\begin{array}{l}\text { Más de } \\
3 \text { horas }\end{array}$ & $\begin{array}{l}\text { hasta } \\
\text { al día }\end{array}$ & & $\begin{array}{l}3 \text { hasta } 4 \\
\text { as al día }\end{array}$ & $\begin{array}{c}\text { Más de } 4 \\
\text { horas al día }\end{array}$ & Valor-p \\
\hline Cs. Ing. & 0.76 & & $6.06 \%$ & 18.1 & $3 \%$ & & $4.24 \%$ & $50.76 \%$ & \\
\hline Cs. Salud & 2.68 & & $5.36 \%$ & 12.9 & $5 \%$ & & $3.66 \%$ & $55.36 \%$ & 0.218 \\
\hline Humanidades & 1.31 & & $11.11 \%$ & 16.3 & $4 \%$ & & $3.53 \%$ & $47.71 \%$ & \\
\hline
\end{tabular}




\section{DISCUSIÓN}

Un primer aspecto para discutir tiene relación con las limitaciones de esta investigación, la primera de ellas es el hecho que la muestra fue intencional por sujeto voluntario y no al azar por lo que, en rigor, los resultados obtenidos se limitan a la muestra estudiada. Además, no fue posible evaluar una muestra de estudiantes que comprendiera todo el territorio nacional, limitándose la muestra al área comprendida entre las regiones de Valparaíso y Bio-Bio (incluida la región Metropolitana). No obstante, estas limitaciones, el hecho de haber evaluado estudiantes de 21 de las 55 universidades existentes en Chile y contar con la participación de 509 sujetos, un número mucho mayor al mínimo muestral determinado estadísticamente, se puede considerar que estos resultados son una aproximación aceptable a la que se podría encontrar en un estudio con muestreo al azar con cobertura nacional. A continuación, se procederá a discutir cada uno de los aspectos analizados en las tablas de resultados.

Los estudiantes de la muestra manifestaron una mejor aproximación al concepto de reputación digital que al concepto de identidad digital. No obstante, ambos aspectos no son de gran preocupación para ellos, por lo que no utilizarían tiempo enfocados a su gestión, tornándose lógico el que tampoco suelan buscarse a sí mismos en Internet para saber qué información respecto de ellos está disponible a todo el mundo. Lo que se puede explicar, acorde a lo concluido por Castañeda et al. (2012), respecto a que, si bien los estudiantes se preocupan de la imagen que proyectan de sí mismos en Internet, esta inquietud es solo de carácter social inmediata, desconociendo la influencia que pueda tener en ellos. En el caso particular de los estudiantes de Ciencias de la Ingeniería, donde un grupo considerable de ellos (31.06\%) asociaba el concepto de reputación digital con la autogestión en red de los conocimientos sobre TIC, explicaría el motivo por el cual fueron el grupo que presentó la mayor preocupación por su reputación digital. Su foco, por tanto, estaría puesto más en la gestión que en la interpretación que otros hacen de la información que ellos dejan en la red.

En cuanto a la similitud entre su yo presencial y su yo digital es relevante el hecho que los estudiantes no hacen gran diferencia entre ellos; cumpliéndose la premisa que sea ya sea online u offline, lo fundamental para los individuos es el habitar un espacio (Belli y De Eugenio, 2014). Pudiéndose inferir que la expresión de su personalidad y su vida a partir de la información disponible en Internet es un reflejo de su propia realidad, lo que es coincidente con los resultados obtenidos en universitarios españoles, los cuales manifiestan que sí creen que son ellos mismos en las redes sociales, mostrando una correspondencia entre su yo real y el de la Web (Castañeda et al., 2012). Este hecho dista de la creencia establecida, en la cual se señala que los individuos presentan un yo en internet diferente a lo que es su vida presencial, lo cual pudiese ser cierto en generaciones anteriores. Quienes se han formado con la presencia de Internet en sus vidas, no verían la necesidad de crear un yo digital que difiera de su yo presencial, es decir, los sujetos estudiados muestran en los medios digitales lo que efectivamente están viviendo, esto coincide con los planteamientos de Sami Cöteli et al. (2019).

Este posible cambio de paradigma nos debe hacer replantear el nivel de confianza respecto a la información que encontramos de los más jóvenes en el plano digital. De hecho, Soomro et al. (2019) en su estudio abordan la necesidad que presentan los individuos de estar conectados en todo momento, como una constante en estudiantes universitarios, coincidiendo con nuestra investigación. Por otro lado, Oviedo-Trespalacios et al. (2019) puntualizan que dicha comunicación constante en medios digitales se da gracias a la omnipresencia que los teléfonos móviles entregan. En la muestra estudiada los sujetos declararon que superan las cuatro horas diarias de dedicación activa en la red, usando preferente la internet del celular o de su hogar, situando a los teléfonos móviles por sobre otros dispositivos de conexión, lo cual es consistente con los resultados de la encuesta de conectividad y uso de Internet del año 2017 (Subsecretaria de Telecomunicaciones, 2018) y a los estudios que han realizado en esta materia Ruiz-Palmero et al. (2016), Barrios et al.( 2017) y Li y Lin (2019).

En cuanto a las actitudes frente a la identidad digital en relación con las interacciones, es interesante ver que, a pesar del alto uso del teléfono móvil, la comunicación presencial para ellos es la primera opción cuando se requiere conversar temas de relevancia según su propio criterio. Pese a sentirse cómodos tanto en el espacio virtual como en el presencial. En cuanto a la segunda opción, se producen leves cambios por área de formación, mientras que para los estudiantes de Humanidades es la videoconferencia, para los estudiantes de Ciencias de la Salud y Ciencias de la Ingeniería es el correo electrónico el canal de comunicación más usado. El orden en la secuencia de prioridades presenta una influencia en relación con el área de formación, aun cuando no existan diferencias significativas en una misma alternativa, según área. Estas diferencias se pueden deber a la influencia de los propios procesos de aprendizaje; así un estudiante de Humanidades aprende a valorar la expresión del lenguaje verbal y no verbal, lo cual se da tanto en la conversación presencial como en la videoconferencia. Por otro lado, los estudiantes de Ciencias de Ingeniería desarrollan habilidades de razonamiento donde la expresión escrita suele ser más relevante, por lo que el correo electrónico y el mensaje de texto se presentan como alternativas en las que se sienten más cómodos. A nivel comunicativo, 
a pesar del gran desarrollo de los medios de comunicación digital, las videoconferencias y correos electrónicos no son elección prioritaria, aunque con la multiplicidad de funciones de los teléfonos celulares, van ganando espacio y dependencia (Liu et al., 2019; Li y Lin, 2019).

En cuanto al ningufoneo (phubbing) entendido como el mirar y usar un dispositivo para conectarse a internet mientras se está interactuando con otros, la conducta declarada por los estudiantes de la muestra nos evidencia que este de tipo de acciones está vinculada al grado de intimidad, cercanía o importancia que le asignan al o los individuos con quienes están interactuando. Es por esto que, cuando están con sus parejas o familia, evitan interactuar con otros desde su celular, tornando esta acción en algo menos frecuente que cuando están solos o con amigos e incluso mientras están participando en clases, existiendo una clara secuencia de prioridades para ellos. En el estudio realizado por Vanden et al. (2019) se estableció que las personas perciben menos intimidad en la conversación cuando la pareja usaba el celular y que en el $30 \%$ de las conversaciones donde las personas sí utilizaban el teléfono, incluían a su interlocutor en la interacción por medio de la acción de compartirle la pantalla. Esto nos lleva a pensar que esta nueva generación ha comenzado a ver el no uso de los teléfonos una forma de expresar al otro $u$ otros lo importancia que representan para ellos y, en caso de emplear sus teléfonos, utilizarlos como un medio de interacción más que una forma de aislamiento. Es decir, se podría considerar en esta nueva generación al ningufoneo como una forma de violencia al mostrar al otro que no lo ve como un individuo importante o que merezca plena atención, más que el resultado de una adicción.

Respecto de los planos de expresión de su identidad, los estudiantes se sientan tan libres de ser en la presencialidad como en la en la virtualidad, reforzándose la concepción que para ellos no es necesaria la dualidad del yo, lo cual coincide con lo expuesto por Belli y De Eugenio (2014). Esto es consistente con el hecho que no hagan diferencia entre su yo en medios digitales y en la presencialidad. No obstante, cabe hacer la salvedad de que, al ser jóvenes y sin experiencia laboral, esta constante de mostrarse tan transparentemente puede cambiar en el tiempo. Ello puesto que, para poblaciones de adultos jóvenes profesionales, la excesiva transparencia lejos de dar confianza genera desconfianza; buscando el equilibrio entre sus identidades privadas y profesionales (Brandtzaeg y Chaparro, 2019).

Finalmente, se puede indicar que el área de formación no presenta una gran influencia en todas las dimensiones que fueron estudiadas, su impacto se centra en aspecto que pueden ser considerados conceptuales o como el resultado del aprendizaje producto de su formación profesional. Esto explicaría la razón de que se encontraran diferencias significativas en relación con el concepto de reputación digital y la forma en que priorizan el medio preferente de interacción con el otro, así como también el mayor uso del PC de escritorio por parte de los estudiantes de Humanidades, quienes requieren pantallas más grandes que faciliten la lectura extensa de textos y que los celulares no ofrecen. Los estudiantes de Ciencias de la Salud y Ciencias de la Ingeniería, en cambio, sus lecturas son más bien consultivas la gran mayoría de las veces, por lo que el celular representa una útil alternativa. Esto, unido a que los teléfonos inteligentes, como objetos de aprendizaje móvil, constituyen un recurso digital indispensable al poner a disposición del estudiante, desde cualquier lugar y tiempo, una amplia gama de alternativas educativas que los transforman en una opción válida para el aprendizaje autónomo. Coincidiendo con las preferencias demostradas por los estudiantes de la Universidad Técnica del Norte de Ecuador en el estudio de Basantes et al. (2017) y de López y Silva (2016).

\section{CONCLUSIONES}

De acuerdo a los resultados de este estudio y de su discusión, de la comparación con resultados de otros autores y del detallado análisis presentado, se pueden extraer las siguiente conclusiones: 1) los estudiantes encuestados no hacen gran diferencia entre su yo presencial y su yo digital, sintiendo igual grado de libertad de expresar su individualidad tanto en su presencialidad como en la virtualidad; 2) manifiestan una gran necesidad de estar conectados a la red, lo que favorece el uso de los teléfonos móviles y la conectividad a través del servicio de Internet de este; 3 ) la presencialidad en la interacción con los otros sigue siendo relevante para ellos cuando el tema es trascendente, aunque los medios digitales de comunicación van ganando un mayor espacio; 4) el área de formación presenta una influencia en cuanto a la interpretación de conceptos y a la priorización de medios de interacción con otros; 5) la separación entre digital y lo presencial a diferencia de generaciones anteriores es mucho menor, lo que proyectivamente podría romper con el concepto entre lo real y virtual; y 6 ) la muestra estudiaba manifiesta un bajo nivel de ningufoneo, su intensidad está asociada al grado de intimidad o relevancia que le da el individuo al otro u otros con las cuales esta interactuando.

\section{REFERENCIAS}

Abdallah, S., Digital Identity and Reputation in a Virtual Practice Network-Insights Using Linguistic Features. http://dx.doi.org/10.4018/ljeC.2018100101, International Journal of e-Collaboration (IJeC), 14(4), 1-23. (2018). 
Barrios, I., Vargas, M., Echeverría, J., García, J., Torales, J., Uso de Tecnologías de la Información y Comunicación para investigación en estudiantes de medicina paraguayos, Revista Cubana de Educación Médica Superior [Internet], 31(4). (2018).

Basantes, A., Naranjo, M., Gallegos, M., Benítez, N., Los Dispositivos Móviles en el Proceso de Aprendizaje de la Facultad de Educación Ciencia y Tecnología de la Universidad Técnica del Norte de Ecuador. http://dx.doi.org/10.4067/S071850062017000200009Cabero, Formación Universitaria, 10(2), 79-88. (2017).

Belli, S., De Eugenio, G. Emotional Practices and processes of subjectivation in digital society: the case of online suicides. http://dx.doi.org/10.5565/rev/qpsicologia.1181, Quaderns de Psicología, 16(2), 57-72. (2014).

Brandtzaeg, P. B., Chaparro-Domínguez, M. Á., From youthful experimentation to professional identity: Understanding identity transitions in social media. http://dx.doi.org/10.1177/1103308819834386, YOUNG, 28(2), 157-174. (2020).

Cabero J., Llorente, M., La aplicación del juicio de experto como técnica de evaluación de las tecnologías de la información y comunicación (TIC), Revista de Tecnología de Información y Comunicación en Educación, 7(2), 11-22. (2013).

Castañeda, L., y Camacho, M., Desvelando nuestra identidad digital. http://dx.doi.org/10.3145/epi.2012.jul.04, El Profesional de la Información, 21(4), 354-360. ( 2012a).

Castro, P., González -Palta, I., Percepción de Estudiantes de Psicología sobre el Uso de Facebook para Desarrollar Pensamiento Crítico. DOI:10.4067/S0718- 50062016000100006. Revista Formación Universitaria, 9(1), 45-56. (2016).

Çöteli, S., The impact of new media on the forms of culture: digital identity and digital culture. http://dx.doi.org/10.29333/ojcmt/5765, Online Journal of communication and media technologies. 9(2). (2019).

Feher, K., Digital identity and the online self: Footprint strategies-An exploratory and comparative research study. http://dx.doi.org/10.1177/0165551519879702, Journal of Information Science. 1-14. (2019).

Garcia-Garcia, J., Reding-Bernal, A., López-Alvarenga, J., Cálculo del tamaño de la muestra en investigación en educación médica, http://dx.doi.org/10.1016/S2007-5057(13)72715-7. Investigación educ. médica [online]., 2(8),217-224. (2013).

Georges F., A l'image de l'Homme: cyborgs, avatars, identités numériques. http://dx.doi.org/10.3917/tdm.018.0136, Le Temps des médias, $1, \mathrm{n}^{\circ} 18$, p. 136-147. (2012).

Hilt, J. A., Dependencia del celular, hábitos y actitudes hacia la lectura y su relación con el rendimiento académico, https://doi.org/10.17162/au.v9i3.384, Apuntes Universitarios, 9(3).16. (2019).

Holicza, P., Kadëna, E., Smart and Secure? Millennials on Mobile Devices. http://dx.doi.org/10.7906/indecs.16.3.10, Interdisciplinary Description of Complex Systems: INDECS, 16(3-A), 376-383. (2018).

Kietzmann, J., Hermkens, K., McCarthy, I., Silvestre, B., Social media? Get serious! Understanding the functional building blocks of social media. http://dx.doi.org/doi.org/10.1016/j.bushor.2011.01.005, Business horizons, 54 (3) (00076813), 241251. (2011).

Kundapur, R., Narasimha, H. H., Baisil, S., Badiger, S., Assessment of Smartphone Addiction among adolescents in a University, Indian Journal of Community Health, 32(1), 161-163. (2020).

Kurek, A., Jose, P. E., Stuart, J., Discovering unique profiles of adolescent information and communication technology (ICT) use: Are ICT use preferences associated with identity and behaviour development? http://dx.doi.org/10.5817/CP2017-4-3, Cyberpsychology: Journal of Psychosocial Research on Cyberspace, 11(4). (2017).

Li, L., Lin, T. T., Over-coLinnected? A qualitative exploration of smartphone addiction among working adults in China. http://dx.doi.org/10.1186/s12888-019-2170-z, BMC psychiatry, 19(1), 186. (2019).

Liu, R. D., Wang, J., Gu, D., Ding, Y., Oei, T. P., Hong, W., Zhen, R. Li, Y., The Effect of Parental Phubbing on Teenager's Mobile Phone Dependency Behaviors: The Mediation Role of Subjective Norm and Dependency Intention. http://dx.doi.org/10.2147/PRBM.S224133, Psychology Research and Behavior Management,12, 1059 -1069. (2019).

López, F., Silva, M. M., Factores que inciden en la aceptación de los dispositivos móviles para el aprendizaje en educación superior. http://dx.doi.org/10.15581/004.30.175-1955, Estudios sobre Educación, 30, 175-195. (2016).

Magro, C., Educación Conectada en Tiempos de Redes. $1^{\circ}$ Ed.51-58, Ministerio de Educación, Cultura y Deporte. España. (2016).

Ministerio de Educación. Directorio Instituciones Educación superior. Subsecretaria de Educación Superior, Chile. (2020)

Oviedo-Trespalacios O, Nandavar S, Newton J, Demant D, Phillips J., Problematic Use of Mobile Phones in Australia...Is It Getting Worse? http://dx.doi.org/10.3389/fpsyt.2019.00105, Frontiers in Psychiatry. 10 (105), 0-0 (2019).

Ruiz-Palmero, J., Sánchez-Rodríguez, J., Trujillo-Torres, J.M., Utilización de Internet y dependencia a teléfonos móviles en adolescentes. http://dx.doi.org/10.11600/1692715x.14232080715, Revista Latinoamericana de Ciencias Sociales, Niñez y Juventud, 14(2), 1357-1369. (2016).

Soomro, K. A., Zai, S. A. Y., Hina, Q. A., Investigating the impact of university students' smartphone addiction on their satisfaction with classroom connectedness. http://dx.doi.org/10.1007/s10639-019-09947-7, Education and Information Technologies, 24(6), 3523-3535. (2019).

Stuart, J., Kurek, A., Looking hot in selfies: Narcissistic beginnings, aggressive outcomes?. http://dx.doi.org/10.1177/0165025419865621, International Journal of Behavioral Development, 43(6), 500-506. (2019). 
Subsecretaría de Telecomunicaciones. IX Encuesta Acceso y Uso de internet. Santiago, Chile. (2018).

Vanden Abeele, M. M. P., Hendrickson, A. T., Pollmann, M. M. y Ling, R., Phubbing Behavior in Conversations and its Relation to Perceived Conversation Intimacy and Distraction: An Exploratory Observation Study. http://dx.doi.org/10.1016/j.chb.2019.06.004, Computers in Human Behavior, 100, 35-47. (2019).

Williams, S., Fleming, S., Lundqvist, K., Parslow, P., This is me: Digital identity and reputation on the internet. In Digital identity and social media. http://dx.doi.org/10.4018/978-1-4666-1915-9.ch008. IGI Global. (pp. 104-117). (2013).

Yu, L., Li, H., He, W., Wang, F. K., Jiao, S. A meta-analysis to explore privacy cognition and information disclosure of internet users. http://dx.doi.org/10.1016/j.ijinfomgt.2019.09.011, International Journal of Information Management, 51, 102015. (2020). 